\title{
Lia Giancristofaro, Valentina Lapiccirella Zingari, Patrimonio culturale immateriale e società civile
}

Aracne, Roma, 2020

Pietro Clemente

\section{CpenEdition}

\section{Journals}

Edizione digitale

URL: https://journals.openedition.org/aam/3617

ISSN: 2038-3215

\section{Editore}

Dipartimento Culture e Società - Università di Palermo

Notizia bibliografica digitale

Pietro Clemente, «Lia Giancristofaro, Valentina Lapiccirella Zingari, Patrimonio culturale immateriale e società civile», Archivio antropologico mediterraneo [Online], Anno XXIII, n. 22 (2) | 2020, online dal 31 décembre 2020, consultato il 20 juillet 2021. URL: http://journals.openedition.org/aam/3617

Questo documento è stato generato automaticamente il 20 juillet 2021.

\section{(c) (1) () $\Theta$}

Archivio antropologico mediterraneo è distribuita con Licenza Creative Commons Attribuzione - Non commerciale - Non opere derivate 4.0 Internazionale. 


\section{Lia Giancristofaro, Valentina} Lapiccirella Zingari, Patrimonio culturale immateriale e società civile

Aracne, Roma, 2020

Pietro Clemente

NOTIZIA

LIA GIANCRISTOFARO, VALENTINA LAPICCIRELLA ZINGARI, Patrimonio culturale immateriale e società civile, Aracne, Roma, 2020.

ISBN 9788825531459 
Dalla Premessa di Pietro Clemente.

«Ricordi. Sono stato coinvolto dal Mibact nel 2007/8 nelle iniziative che il Ministro Rutelli volle proporre in occasione della adesione dell'Italia alla Convenzione sul Patrimonio Culturale Immateriale che era stata definita dall'Unesco nel 2003. Una convenzione che aveva modificato l'orientamento precedente: infatti vi fu dapprima una bozza di convenzione sulla 'salvaguardia del folklore', e in seguito la discutibile pratica dei 'Capolavori viventi', che rischiava di trasformare il patrimonio in una hit parade. Con la Convenzione del 2003 si avviava una complessa pratica culturale e giuridica tesa a valorizzare le comunità portatrici e protagoniste di culture diverse con l'intenzione di porre tali culture all'attenzione di una grande

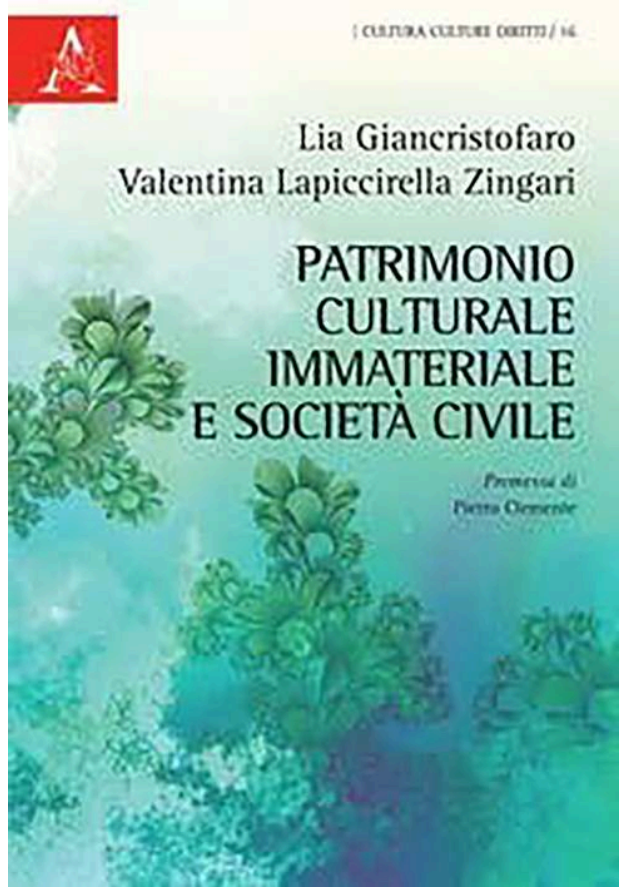
comunità internazionale e di valorizzarle come importanti per la vita delle popolazioni, per il valore del loro passato capace di diventare risorsa di un futuro 'diverso'. Il Ministro nominò una commissione (Comitato scientifico per la valorizzazione delle tradizioni italiane) fatta quasi interamente di studiosi e operatori dell'ambito demo-etno-antropologico, della quale feci parte (la presiedeva Paolo Apolito), che cercò di avviare un modo italiano di interpretare la convenzione. Un modo pensato e condiviso tra studi antropologici, funzionari ministeriali, associazioni del settore (io rappresentavo SIMBDEA, la Società Italiana per la Museografia e i Beni Demo Etno Antropologici). Un modo che sembrava giusto. Ricordo che la prima idea fu quella di favorire le candidature di rete e non quelle singole, di sollecitare non il narcisismo delle comunità ma il valore di appartenenza a una rete internazionale di comunità, basata sulla diversità delle culture: una rete che all'origine nasceva per favorire il dialogo tra popoli e culture, per contrastare la guerra e la distruzione dei patrimoni. Nella convenzione del 2003 c'erano temi nuovi per il mondo del patrimonio. Tra questi il concetto di salvaguardia, un concetto nuovo rispetto a quelli in uso nelle nostre Sovrintendenze. La salvaguardia non è conservazione ma passaggio di un tratto culturale attraverso le generazioni con adattamenti e trasformazioni che lo rendano sempre vivo ed attuale. Un altro grande tema è quello del Patrimonio Culturale Immateriale (in Italia PCI in area anglosassone ICH Intangible Cultural Heritage) che fu e resta assai discusso, da un lato per ragioni di chiarezza concettuale, dall'altro per difficoltà di connessione con il Codice dei beni culturali e del paesaggio (2004). Tale codice è la principale legge italiana sul patrimonio, che prevede quasi esclusivamente un patrimonio 'materiale'. Un ulteriore tema di grande rilievo è quello delle comunità che diventano protagoniste sia della richiesta di riconoscimento, sia della salvaguardia del bene culturale. Novità rilevante e strategica: quasi tutta la produzione di valore delle organizzazioni internazionali dagli anni 2000 si basa sul protagonismo dei soggetti 'portatori'. Su questi temi la comunità scientifica si interroga ancora oggi e discute: per alcuni sono temi nuovi e trainanti che richiedono anche una forte trasformazione 
dell'antropologia, per altri invece sono imposti dall'alto e sottoposti ai giochi dei poteri politici locali, nazionali e internazionali.

3 Ma ben presto. Torniamo al Comitato Scientifico nominato dal Ministro Rutelli. L'attenzione del governo alla Convenzione era cominciata con una 'buona pratica'. Ma ben presto il governo cadde (l'ultimo dell'Ulivo) e il Comitato nominato da Rutelli scomparve come neve al sole, il nuovo Ministro Bondi non pensò nemmeno per un attimo né alla Convenzione né alla nostra commissione, lo spazio Unesco finì per essere terreno di contese in una sfera legata a governo e sottogoverno, senza più alcun ricorso agli antropologi, che sono - in quasi tutti i paesi che condividono la Convenzione - tra i principali consulenti e interpreti dell'andamento e dei cambiamenti della Convenzione, ma non in Italia. Nel tempo all'interno del Mibact (ora Mibac) si sono formate delle Commissioni di lavoro interne al ministero, con la funzione di esaminare le candidature, di guidarle in qualche caso, con un ruolo significativo dell'ICDEA (Istituto Centrale per la Demo-Etno-Antropologia) mentre resta quasi ai margini dell'attività l'elefantiaco Consiglio Nazionale Unesco Italia. Una situazione politico-amministrativa davvero gracile e inadeguata a un ruolo dell'Italia nel consesso internazionale, o anche solo nel monitoraggio e interpretazione del proprio patrimonio culturale. Non c'è dubbio che siamo in ritardo su questo fronte. Questo libro vuole avere anche lo scopo di fare passi avanti cercando di formare i giovani e di far entrare il patrimonio culturale immateriale nel loro repertorio di conoscenze e di capacità analitiche. Forse per il prevalere della storia dell'arte, dell'archeologia e dell'architettura nella gestione del patrimonio, forse per lo scarso investimento politico dei governi sulla cultura e le organizzazioni internazionali che la coinvolgono, possiamo considerare davvero poco aggiornata l'attività statale in questo ambito.

4 La convenzione. Per chi si occupa del patrimonio come antropologo culturale la Convenzione resta un importante punto di riferimento per aggiornare e internazionalizzare concetti, pratiche, modi di collaborazione con le realtà locali e con le reti nazionali che rappresentano, almeno in parte, la società civile. La convenzione del 2003 ha ormai avuto una lunga fase di sperimentazione, in cui ci sono stati anche aggiornamenti e innovazioni, dialoghi internazionali, scambi tra antropologi di vari paesi del mondo, oltreché riconoscimenti Unesco, sempre piuttosto discussi, anche a ragione, ma per lo più perché la scarsa conoscenza della Convenzione fa sì che la Lista del PCI sia vista come una 'hit parade' e non come una costellazione di comunità culturalmente specifiche che hanno chiesto e ottenuto un riconoscimento. Nel tempo, per chi segue le problematiche politico-culturali del patrimonio, la normativa possibile degli Stati in questo ambito si è arricchita di innesti. Tra questi in particolare la Convenzione di Faro [...] che approfondisce il tema delle 'comunità patrimoniali', o 'comunità di eredità'. Si è formato uno spazio intellettuale ed esperienziale interdisciplinare fatto di giuristi, antropologi, esperti della documentazione, esperti di varie tipologie di saperi pratici, educatori, esperti di territorio e sviluppo locale, che cerca di costruire un lessico capace di dare voce alla pluralità delle differenze culturali e scambiare esperienze internazionali. Le due autrici di questo volume fanno parte di questo mondo di nuovi esperti/e, antropologhe/i aperte/i a nuove esperienze e dialoghi, che hanno seguito gli incontri internazionali, che conoscono le pratiche di candidatura delle comunità, che hanno vissuto dibattiti, contraddizioni, problemi e delusioni di un contesto internazionale difficile. $\mathrm{E}$ hanno vissuto anche le esperienze fatte dagli antropologi per seguire collaborativamente i contesti locali, e le comunità che si attivano per i riconoscimenti. In particolare Giancristofaro e Zingari sono attive 
in una richiesta di salvaguardia urgente per la festa di San Domenico di Cocullo, in Abruzzo, e dei saperi dei 'serpari'. La salvaguardia urgente è un tipo di richiesta che impegna l'UNESCO nell'intervento di sostegno alle comunità che sono a rischio di sopravvivenza, mentre per le altre candidature della Lista l'UNESCO non interviene, anzi insiste perché siano le istituzioni politico-amministrative che sostengono la candidatura a intervenire anche finanziariamente nella valorizzazione.

5 Si capisce forse, da quanto dico, che la Convenzione UNESCO del 2003 cerca di coniugare la piccola dimensione propria degli studi antropologici (il "villaggio", o il "tratto culturale") con categorie di riconoscimento internazionali, e soprattutto cerca di dare la priorità al protagonismo delle comunità locali richiedenti, non agli esperti né ai rappresentanti politici. Gli 'esperti', 'noi', entriamo in campo oggi come collaboratori delle comunità, al servizio del 'potenziamento' (enpowerment) delle loro esigenze. Un difficile equilibrio, sempre critico, sempre a rischio di difetti.

6 La nozione di patrimonio 'immateriale', così discussa, da molti considerata concettualmente errata, contesa tra diversi campi del sapere, ha avuto tuttavia una forte capacità di rianimare il mondo antropologico dei musei e della cultura materiale, spingendo a vedere sempre il primato dei saperi rispetto a quello delle cose. Accrescendo la possibilità della salvaguardia e anche della riattivazione dei saperi e del saper fare.

7 Il patrimonio e il mondo. Con lo sguardo lungo che ho appreso dagli studiosi del diritto internazionale, ho compreso che la Convenzione, nell'ambito della dimensione generale dell'UNESCO (avviata già dal 1975 per i patrimoni materiali), eredita e potenzia quelle forme di legislazione internazionale universalistiche che cercano di far dialogare $i$ popoli, evitare le guerre e, in caso di guerra, evitare le distruzioni dei patrimoni culturali. Si parte dalle Convenzioni di Ginevra, alla nascita delle Nazioni Unite e poi dell'ONU e quindi dell'UNESCO. Una legislazione difficile in un mondo dominato dalla crisi delle relazioni internazionali e animato da nuove tendenze di chiusura delle nazioni al mondo (sovranismo). Il rilievo dell'Unesco è stato evidente in occasione degli schieramenti politici interstatali che portarono al riconoscimento come stato membro della Palestina, con reazioni assai negative di USA e Israele. È evidente, almeno a me, che l'UNESCO è uno dei campi dove a livello mondiale si lotta per la democrazia e contro la guerra, e che il 'patrimonio' è uno dei modi e dei terreni di contesa. Il patrimonio è uno dei modi nuovi perché passa per le differenze culturali e il dialogo tra mondi di riferimento traversati da etiche, religioni, forme di vita, e che quindi corregge anche l'universalismo di matrice occidentale che ispira molte organizzazioni internazionali.

8 Società civile. Dall'esperienza UNESCO nasce in gran parte anche la riflessione sul ruolo della ricerca antropologica nella società globale, in cui si danno nuove prospettive di protagonismo ai soggetti locali. Nasce la riflessione sul ruolo dei REI (registro delle eredità Immateriali) come aspetto principale delle attività PCI nelle Regioni che li adottano (per ora Lombardia, Sicilia), sul rapporto tra valori universali e differenze culturali. Una antropologia che non si limita ad analizzare e denunciare, ma che ha una funzione fondamentalmente collaborativa, in cui analisi e interpretazione sono legate alla consapevolezza e alla crescita delle comunità, in cui la critica politica cerca di diventare dialogo, pratica di confronto, programmazione collaborativa. Il concetto di 'società civile' che campeggia come secondo elemento forte nel titolo del libro, rappresenta in buona parte questa attività. Ed è in questo ambito che è stato possibile 
elaborare sia nuove immagini di cosa sia oggi una comunità, di quale sia l'atteggiamento dell'antropologo verso le comunità, cercando di capire il nesso tra il patrimonio e lo sviluppo locale, il patrimonio e la democrazia rappresentativa. Lo stesso concetto di patrimonio introdotto il Italia nel dibattito degli anni Novanta ed entrato nella legislazione con il Codice del 2004, sostituendo o affiancandosi a quello di beni culturali, ha avuto nella normativa della tutela del patrimonio una capacità di sviluppare attitudini contestuali, insiemi, allargamenti, contro l'approccio estetico ed esclusivo del mondo dei beni una volta detti 'belle arti'.

Sono temi che si connettono con uno spazio sociale, quello della società civile, concetto che nasce dentro la filosofia dell'Ottocento nell'idealismo tedesco di Hegel, che vide famiglia, società civile, stato, come scale di articolazione e di complessità delle società, dal semplice al complesso. Una tematica ripresa da Antonio Gramsci allo scopo di descrivere la articolazione sociale, nella prospettiva politica di alleanze e analisi stratificate e minuziose. Infine ripresa e ridiscussa in un contesto internazionale per indicare i fattori positivi e progressivi dell'azione sociale organizzata dal basso.

Il patrimonio è uno spazio di azione sociale in cui operano tanti diversi profili di ricercatori. Esso viene visto talora come inadeguato di fronte alla necessità, per alcuni unica e assoluta, di critica politica del sistema capitalistico. In questa ottica il patrimonio diventa nient'altro che una forma di travestimento ulteriore di un capitalismo (o neoliberismo) che intende ridurre il mondo a consumo e subordinazione. Da quel che ho detto sembra evidente che una critica sociale radicale e esterna all' analisi locale, non fa che ridurre la critica alla ideologia, e tendenzialmente esclude l'azione concreta e attiva dell'antropologo. Vivere, lottare, agire nella società civile appare invece, a chi pratica questo ambito, il luogo vero del cambiamento e della azione pratica, anche limitatamente a un settore specifico e al modo antropologico di fare ricerca legato alle piccole dimensioni e ai grandi nodi concettuali.

11 Questo libro propone un modo sistematico di vedere la legislazione sul patrimonio culturale immateriale come parte della realtà giuridica del nostro paese, una parte in ombra nell'azione politico-amministrativa pubblica, e scarsamente affidata alla competenza antropologica. Un campo normativo ricco di ideali universalisti e al tempo stesso pluralisti e polifonici, che diventa ambito di operatività politica e intellettuale capace di potenziare una cultura democratica e partecipativa, e di mettere a disposizione del futuro risorse ed esperienze umane di diverse comunità del mondo che possono essere anche un fattore di salvezza e di riflessività critica per le società e le generazioni di domani». 